FORMATION Formation emploi

Revue française de sciences sociales

99 | juillet-septembre 2007

Les usages sociaux de la compétence

\title{
Certification européenne : rapprochement des structures nationales de formation?
}

European qualifications: Should national vocational training structures be made more uniform?

Europäische Zertifizierung: Angleichung der einzelstaatlichen

Ausbildungssysteme?

Joachim Haas et Maurice Ourtau

\section{OpenEdition}

Journals

Édition électronique

URL : http://journals.openedition.org/formationemploi/1511

DOI : 10.4000/formationemploi.1511

ISSN : 2107-0946

Éditeur

La Documentation française

Édition imprimée

Date de publication : 1 juillet 2007

Pagination : 123-137

ISSN : 0759-6340

Référence électronique

Joachim Haas et Maurice Ourtau, «Certification européenne : rapprochement des structures nationales de formation? ». Formation emploi [En ligne], 99 | juillet-septembre 2007, mis en ligne le 30 septembre 2009, consulté le 30 octobre 2020. URL : http://journals.openedition.org/formationemploi/ 1511 ; DOI : https://doi.org/10.4000/formationemploi.1511 


\title{
étrangers
}

\section{Certification européenne : rapprochement des structures nationales de formation?}

\author{
par Joachim Haas et Maurice Ourtau*
}

\section{L'imposition des normes européennes est confrontée à la fois au poids des structures nationales et à la distribution traditionnelle des rôles entre les acteurs; pour autant, assiste-t-on à une remise en question des régimes nationaux?}

En vertu du principe de libre circulation de la maind'œuvre, et dans le but de favoriser la reconnaissance des qualifications au sein de la Communauté européenne, de nouvelles modalités de certifications apparaissent, et avec elles de nouvelles instances de régulation chargées d'en contrôler les applications nationales.

Comment trois pays (Allemagne, Royaume-Uni et France), confrontés à la transposition d'une norme de ce type, édictée par une autorité supranationale, ontils envisagé et mis en œuvre des solutions particulières ?

Dans quelle mesure les solutions adoptées par chacun des pays disposant, pour la gestion de leurs systèmes de formation professionnelle, d'une marge de manœuvre au titre du principe de subsidiarité ${ }^{1}$

${ }^{1}$ Ce principe a été introduit dans le droit communautaire par le traité de Maastricht (1992). Il consiste à réserver uniquement à l'échelon supérieur, la Communauté européenne (CE), ce que l'échelon inférieur, les États membres de la $\mathrm{CE}$, ne pourrait effectuer que de manière moins efficace. Il ne s'applique qu'aux questions relevant d'une compétence partagée entre la Communauté et les États membres, susceptibles de poser des problèmes d'attribution. En matière de formation professionnelle, la Communauté est compétente pour adopter des «mesures » dans ce domaine, mais elle doit respecter la "responsabilité des Etats membres pour le contenu et l'organisation de la formation professionnelle ». En outre, l'harmonisation des législations est expressément exclue.
Joachim Haas est chargé d'études Céreq au Lirhe (Laboratoire interdisciplinaire de recherches sur les ressources humaines et l'emploi), centre associé au Céreq de Toulouse, université de Toulouse I. Il est diplômé en sociologie et sciences politiques des universités de Constance, Paris et Heidelberg. Ses recherches portent sur des systèmes d'éducation et d'emploi dans une perspective comparatiste et sur la sociologie du travail dans l'aéronautique et l'espace. II a notamment publié : Haas J. (2005), "The Situation in Industry and the Loss of Interest in Science Education», European Journal of Vocational Training, 35, 2, pp. 21 27. Haas J. (2007), "Mecanic vallée : Interactions entre système productif local et formation », Formation Emploi $n^{\circ} 97$, pp. 9-21.

Maurice Ourtau est économiste, ingénieur de recherche CNRS au Lirhe, université de Toulouse I. II a participé à plusieurs observatoires du Céreq, contribué à la conception d'outils d'analyse régionale des relations formationemploi (dispositif ARGOS), réalisé plusieurs travaux sur la négociation et la création des diplômes (fonctionnement des Commissions professionnelles consultatives, rapport coordonné par Lucie Tanguy : Quelles formations pour les employés et les ouvriers en France ? (1991) ; Les diplômes professionnels en Allemagne et en France, MöbusVerdier (Éds), L'Harmattan (1997). Plus récemment, il a 
travaillé sur l'emploi et le travail non qualifié, en contribuant à l'ouvrage : Le travail non qualifié, permanences et paradoxes, sous la direction de D. Méda et F. Vennat, La Découverte, 2004, ainsi que sur l'évolution des qualifications dans le secteur aéronautique.

qui prévaut dans ce domaine, reflètent-elles leurs caractéristiques structurelles en matière d'éducation, de formation et de certification?

La soumission à une réglementation uniformisée au plan européen génère-t-elle un rapprochement des solutions qui impliquerait l'éloignement, l'abandon partiel des principes à la base des caractéristiques structurelles nationales, ou bien au contraire contribue-t-elle au renforcement de ces principes?

Telles sont les questions abordées dans cet article à partir de l'étude de la normalisation européenne en matière de certification des qualifications des agents de la maintenance aéronautique ${ }^{2}(c f$. encadré $\mathbf{1})$. Nos préoccupations rejoignent celles de travaux antérieurs sur le système français des diplômes, concernant les diplômes professionnels en France et en Allemagne. Philippe Méhaut (1997) concluait, qu'en France, les discours contre la rigidité des diplômes et la référence à des normes alternatives (comme l'accréditation individuelle mise en exergue au niveau européen), avaient peu de chances d'aboutir à la disparition du diplôme. Plusieurs travaux portant sur les comparaisons internationales des systèmes nationaux de formation, de construction et de reconnaissance des diplômes et certifications (Möbus et Verdier, 1997) ont fait état de caractéristiques fortes dans chaque pays.

Ces caractéristiques nationales sont perçues par des acteurs dans le champ de la certification comme une difficulté potentielle aux rapprochements européens (cf. Charraud, 2002).

Un des obstacles essentiels à la standardisation des qualifications serait la grande variété des solutions nationales historiquement retenues et la forte interdé-

\footnotetext{
2 Cette étude bénéficiait d'une convention avec le ministère de la Jeunesse, de l'Éducation et de la Recherche (Direction de l'enseignement scolaire) suite à un appel d'offre sur le thème : "la certification, rôle et place des diplômes professionnels dans l'espace des certifications professionnelles ».
}

pendance de ces solutions avec le contexte systémique national plus large. La persistance de cohérences structurelles nationales particulières, mises en exergue dans les analyses sociétales menées notamment par Maurice, Sellier, Silvestre (1982), laisse augurer une diversité de solutions dans les modalités d'application de la norme.

Des auteurs comme Soskice et Hancké (1997) avancent que la valeur que les acteurs économiques attribuent aux certifications de formation et d'enseignement dépend des modalités nationales d'articulation (qualifiées de "régime») entre plusieurs dimensions que sont les politiques financières (à long ou à court terme), le type de gestion des ressources humaines qui en découle (recherche, immédiate ou non, de réduction des coûts salariaux), le système de relations industrielles plus ou moins conflictuel, et enfin l'appareil de formation professionnelle plus ou moins structuré. Ces auteurs estiment difficile d'effectuer des changements dans une seule des composantes de chaque système national sans en faire autant pour les autres. Dès lors, quelles sont les conséquences d'une normalisation de la qualification des agents de la maintenance aéronautique ( $c f$. encadré $\mathbf{1}$ ), construite en dehors de chacun des systèmes propres aux trois pays retenus et sur lesquels elle devra néanmoins se greffer?

Dans ces conditions de forte prégnance des structures nationales, on peut s'attendre, sinon à un déterminisme strict dans les ajustements opérés, du moins à une certaine dépendance, telle qu'elle est formulée dans les analyses en termes de «sentier» (Foray et David, 1995).

La première partie de cette contribution est consacrée à une analyse comparative ( $c f$. encadré 2 ), au regard de cinq critères classants, des modalités de transposition des règlements européens pour les trois pays. Une seconde partie identifie les positions des acteurs nationaux, afin de rendre compte de quelques-unes des grandes questions en débat lors de l'adoption de ces modalités.

En guise de conclusion sont rassemblés des éléments de réponse à la question introductive sur la tendance à l'atténuation et/ou au renforcement des particularismes nationaux. 


\section{Encadré 1 \\ Les normes JARs/Parts dans la maintenance aéronautique}

Vers la fin des années 60, les avionneurs européens ont incité à la création de normes techniques dans la fabrication des avions afin d'éviter la multiplication de réglementations propres à chacun des pays clients. Un dispositif de normes appelé JAR Uoint Aviation Requirement) a été développé par le JAA Uoint Aviation Authorities), association qui représentait les Autorités nationales européennes de l'aviation. À la fin des années 80 , cette standardisation technique a été complétée par un projet de mise en œuvre des normes et procédures réglementaires communes pour les organisations et la qualification des individus. À la suite de la prise en charge du dispositif JAR par la nouvelle Agence européenne de sécurité aérienne (AESA), qui a remplacé le JAA en 2003, les réglementations communautaires ont été renommées "EASA Parts » et sont directement applicables sans être reprises par des réglementations nationales. Avant le développement des JARs, les autorités de l'aviation civile élaboraient des dispositifs nationaux de gestion des qualifications des mécaniciens au regard d'une réglementation minimale édictée par l'OACl (Organisation de l'aviation civile internationale). Beaucoup de pays, dont l'Allemagne et le Royaume-Uni avaient opté pour un système de licences attribuées par les Autorité nationales à des individus autorisés à exercer certaines tâches particulières (cf. infra APRS). D'autres pays comme la France avaient en revanche préféré la solution de l'agrément des entreprises de maintenance habilitées à désigner elles-mêmes les mécaniciens jugés aptes à l'exercice de ces tâches ( ${ }^{*}$ ).

Trois normes unifiées régissent dorénavant le processus d'attribution de titres et d'accès à certaines activités réglementées qui concernent la maintenance au sol des aéronefs. Selon une convention, établie par les JAA, chaque norme JAR/Part se distingue par un numéro qui est aligné sur la numérotation du code correspondant de l'aviation civile des États-Unis :

- la «JAR/Part-145» détermine les modes d'organisation et de fonctionnement des ateliers de maintenance; elle est entrée en vigueur dès 1994.

- la "JAR/Part-66» définit les conditions de délivrance des certifications pour les agents devant signer l'Approbation pour la remise en service (APRS) de l'avion ou une de ses composantes. Elle établit à cette fin un système de licences (dans le sens de "permis ", c'està-dire donnant le droit à leurs titulaires d'exercer une activité réglementée), structuré en trois niveaux : licences $A, B$ et $C$, correspondant respectivement au mécanicien d'escale, technicien de dépannage et cadre technique. Chaque licence se caractérise par des spécificités sur le plan du contenu de formation, des spécialisations et des expériences requises, des modes de certification (examens, etc.). La licence dite "complète » est obtenue au terme d'une période de formation de base (sanctionnée par la certification nationale de référence) suivie d'un temps de pratique dans une entreprise agréée JAR/Part-145, dont la durée est réduite pour les candidats ayant suivi leur formation de base dans un établissement agréé JAR/Part-147.

Depuis l'entrée en vigueur du dispositif - octobre 2006 - la détention d'une Licence JAR/Part-66 adéquate est obligatoire pour tout personnel délivrant l'APRS en Europe.

- la "JAR/Part-147 » fixe les règles de fonctionnement des organismes de formation habilités à mettre en œuvre des formations respectant la norme "JAR/Part-66 » et à procéder à la certification des candidats. Ce dispositif est aussi entré en vigueur en 2006.

$(*)$ : Sur les raisons de la position française, $c f$. Haas (2006). 


\section{Encadré 2 \\ La démarche méthodologique}

Elle est exploratoire dans la mesure où nous avons été contraints d'établir un fonds d'informations minimum au moyen d'investigations de terrain. La recherche a en effet révélé le caractère discret de la construction du dispositif JARs/Parts.

La démarche est comparative. Les investigations portent sur trois pays - Allemagne, France, RoyaumeUni - et sur plusieurs organismes européens lassociations, AESA - Agence européenne de sécurité aérienne). La juxtaposition des points de vue des ressortissants des trois pays et des représentants de ces organismes vise une meilleure compréhension des débats survenus lors de la construction du dispositif.

La méthode est qualitative. Elle repose sur une vingtaine d'entretiens semi-directifs (de durées variables, d'une à plusieurs heures) menés dans les trois pays auprès d'experts anciens négociateurs et/ou observateurs, d'experts du ministère des Transports, de syndicats de salariés, d'employeurs de la maintenance, d'acteurs du système éducatif impliqués. Une grille d'analyse, communiquée aux interlocuteurs préalablement aux entretiens, scindait les questions en deux grands blocs: d'une part, les grands débats qui ont animé les négociations sur la construction de la norme et le rôle des acteurs; d'autre part, les solutions envisagées pour sa mise en œuvre nationale et les problèmes associés. Quelques-uns ont répondu par écrit.

Enfin, la démarche est historique. Les négociations pour la construction du système JAR/Part-66 se sont échelonnées de 1989 à 1998. Les experts recherchés et interviewés sont donc souvent des "vétérans », à la retraite et/ou difficiles à identifier et à joindre le cas échéant.

\section{DES SOLUTIONS NATIONALES ANCRÉES DANS LES STRUCTURES DE FORMATION EXISTANTES}

Pour chacun des deux niveaux de licence, A et B ( $c f$. encadré 1), en application du principe de subsidiarité défini plus haut, les modalités de la formation professionnelle de base permettant d'accéder au référentiel de certification européen normalisé sont choisies par les pays membres. Nos analyses ont porté sur ces deux catégories A et $\mathrm{B}$ de licence, la licence $\mathrm{C}$ concernant très peu de personnel et étant plus centrée sur le management que sur des tâches techniques ${ }^{3}$.

La caractéristique clé du dispositif Part-66 permettant des solutions nationales réside dans la modularisation du référentiel et des examens associés, répartis en 13 modules pour chacune des deux licences. Les examens peuvent être gérés, à travers des questions à

\footnotetext{
3 Pour une présentation détaillée des négociations et des modalités de mise en œuvre des formations de base aux licences dans chacun de trois pays, $c f$. Haas \& Ourtau (2006).
}

choix multiples $(\mathrm{QCM})^{4}$, par l'Autorité nationale ${ }^{5}$. En même temps, et ceci introduit la possibilité pour des arrangements nationaux, chaque Autorité peut reconnaître («valider ») des diplômes techniques nationaux pour des dispenses partielles ou totales d'examens Part-66. Selon la réglementation européenne, de telles reconnaissances, ou «crédits», devront se justifier par une comparaison détaillée des contenus de la formation considérée avec les modules Part-66. Il est donc tout à fait permis de désigner, éventuellement après aménagement, une formation nationale comme «socle» largement validé sur lequel se greffe la licence européenne.

Les enquêtes exploratoires ont révélé des différences nationales remarquables entre les solutions adoptées

\footnotetext{
${ }^{4}$ L'examen comporte 450 questions pour la licence A et 670 questions pour la licence B (d'un niveau plus difficile).

5 Organisme rattaché au ministère des Transports national, l'Autorité a pour mission de surveiller et garantir la sécurité du transport aérien. En France, la DGAC (Direction générale de l'aviation civile) est chargée de cette mission. Les organismes correspondants sont le CAA (Civil aviation authority) pour le Royaume-Uni et le LBA (Luftfahrtbundesamt) pour l'Allemagne.
} 
pour l'introduction du système des licences. Cinq de ces différences sont traitées ici selon les critères suivants :

- la stratégie des systèmes éducatifs ;

- le spectre des formations nationales prises en compte explicitement par l'Autorité de l'aviation civile pour l'ancrage des standards Part-66;

- les modalités d'aménagement des contenus de formation ;

- les formes de validation;

- le recours aux compétences en matière d'ingénierie de la formation.
Eu égard aux différences nationales quant au niveau des formations retenues comme socles pour les deux catégories de licences A et B (niveau ouvrier qualifié et niveau technicien, respectivement), il convient de distinguer les solutions relatives à chacune de ces catégories de licence.

\section{Les solutions nationales pour la licence catégorie $A$ (niveau ouvrier qualifié) $C f$. tableau 1.}

Tableau 1

Les solutions nationales adoptées pour la licence catégorie $A$

\begin{tabular}{|c|c|}
\hline \multicolumn{2}{|r|}{ France } \\
\hline $\begin{array}{l}\text { Stratégies } \\
\text { de l'Éducation }\end{array}$ & $\begin{array}{l}\text { Adaptation institutionnelle en gardant la double finalité du diplôme, propédeutique et pro- } \\
\text { fessionnalisant. } \\
\text { Maintien de l'hégémonie de l'Éducation nationale sur ce segment du marché de la formation. }\end{array}$ \\
\hline $\begin{array}{l}\text { Spectre } \\
\text { des formations } \\
\text { « socle » }\left(^{*}\right)\end{array}$ & $\begin{array}{l}\text { Relativement étroit - deux formations spécifiques plus deux formations de type reconversion } \\
\text { (militaire, AFPA - Association pour la formation professionnelle des adultes). }\end{array}$ \\
\hline $\begin{array}{l}\text { Contenus, } \\
\text { aménagements }\end{array}$ & $\begin{array}{l}\text { Intégration des éléments du standard européen dans les formations nationales existantes } \\
\text { (CAP - certificat d'aptitude professionnelle - de la spécialitél) à l'occasion de la révision du } \\
\text { diplôme et l'aménagement du référentiel. }\end{array}$ \\
\hline $\begin{array}{l}\text { Validation, } \\
\text { crédits }\end{array}$ & $\begin{array}{l}\text { Validation limitée des contenus du programme du CAP traditionnel ; épreuves et modalités } \\
\text { d'examens distinctes pour les modules spécifiques à la licence. }\end{array}$ \\
\hline $\begin{array}{l}\text { Ingénierie } \\
\text { de formation }\end{array}$ & $\begin{array}{l}\text { Du ressort de l'État, avec transfert de compétences (via une convention entre les deux minis- } \\
\text { tères, Transports et Éducation) de l'Autorité vers l'Éducation nationale qui pilote la négocia- } \\
\text { tion sur l'aménagement des formations au sein des instances de consultation (CPC - } \\
\text { Commission professionnelle consultative) et fait intervenir son corps d'Inspection Générale } \\
\text { pour l'accréditation de ses propres établissements. }\end{array}$ \\
\hline \multicolumn{2}{|r|}{ Allemagne } \\
\hline $\begin{array}{l}\text { Stratégies } \\
\text { de l'Éducation }\end{array}$ & $\begin{array}{l}\text { Adaptation institutionnelle protégeant l'identité de la formation aéronautique spécifique } \\
\text { (large spectre de compétences répondant à la diversité des emplois de la profession). } \\
\text { Volonté de préserver la prépondérance de la formation duale sur ce segment de marché de } \\
\text { la formation. }\end{array}$ \\
\hline $\begin{array}{l}\text { Spectre } \\
\text { des formations } \\
\text { « socle » }\end{array}$ & $\begin{array}{l}\text { Large - outre les deux formations dédiées à la maintenance aéronautique (mécanique, } \\
\text { avionique), vingt-cinq formations connexes sont reconnues par l'Autorité. }\end{array}$ \\
\hline
\end{tabular}


Tableau 1 (suite)

\begin{tabular}{|c|c|}
\hline $\begin{array}{l}\text { Contenus, } \\
\text { aménagements }\end{array}$ & $\begin{array}{l}\text { Deux modalités, selon le type de formation: } \\
\text { (a) formations spécialisées de maintenance aéronautique : intégration de compléments dans } \\
\text { les écoles professionnelles publiques (cursus de } 10 \text { jours). } \\
\text { (b) formations connexes : compléments de type « à la carte » offerts par le marché ; cursus } \\
\text { complémentaires individualisés en fonction du bagage de formation de la clientèle. }\end{array}$ \\
\hline $\begin{array}{l}\text { Validation, } \\
\text { crédits }\end{array}$ & $\begin{array}{l}\text { Système de crédits pour vingt-sept formations réduisant fortement les compléments exigés } \\
\text { pour l'accès à la licence, en fonction de la spécialité }\left({ }^{* *}\right) \text { et de la note obtenue en forma- } \\
\text { tion initiale }\left({ }^{* *}\right) \text {. }\end{array}$ \\
\hline $\begin{array}{l}\text { Ingénierie } \\
\text { de formation }\end{array}$ & $\begin{array}{l}\text { Du ressort des entreprises et des écoles professionnelles publiques (système duall), en colla- } \\
\text { boration avec l'Autorité aéronautique. }\end{array}$ \\
\hline \multicolumn{2}{|r|}{ Royaume-Uni } \\
\hline $\begin{array}{l}\text { Stratégies } \\
\text { de l'Éducation }\end{array}$ & $\begin{array}{l}\text { Adaptation par le biais du marché de la formation et des ses acteurs. Les pouvoirs publics } \\
\text { attribuent des subventions pour soutenir les adaptations. }\end{array}$ \\
\hline $\begin{array}{c}\text { Spectre } \\
\text { des formations } \\
\text { « socle » }\end{array}$ & $\begin{array}{l}\text { Aucune reconnaissance par l'Autorité (au sens de validation), des formations existantes. } \\
\text { Grande diversité de l'offre de formation pour préparer aux épreuves de certification organi- } \\
\text { sées par l'Autorité : } \\
\text { (a) formation Part-66 spécialisée ; } \\
\text { (b) formations dans le cadre de parcours individuels (cours du soir, expérience, reconversion } \\
\text { d'anciens militaires...), soutenues ou non par l'entreprise; } \\
\text { (c) apprentissage. }\end{array}$ \\
\hline $\begin{array}{l}\text { Contenus, } \\
\text { aménagements }\end{array}$ & $\begin{array}{l}\text { Des modalités différentes selon le type d'établissement de formation : } \\
\text { (a) dans des écoles privées spécialisées en aéronautique, création de formations spécifi- } \\
\text { quement Part-66. } \\
\text { (b) ailleurs, révision de la formation aéronautique et intégration des éléments du standard } \\
\text { européen, mais dans des cursus comportant également une dimension plus propédeutique. }\end{array}$ \\
\hline $\begin{array}{l}\text { Validation, } \\
\text { crédits }\end{array}$ & $\begin{array}{l}\text { Aucune validation de formations, pas de système de crédits. } \\
\text { Tous les candidats passent les examens dans des centres gérés ou agréés par l'Autorité de } \\
\text { l'aviation civile. }\end{array}$ \\
\hline $\begin{array}{l}\text { Ingénierie } \\
\text { de formation }\end{array}$ & Du ressort, principalement, des écoles spécialisées bien positionnées sur le marché. \\
\hline
\end{tabular}

$\left(^{*}\right)$ : Formations sur lesquelles sont greffés les compléments de formation.

$(* *)$ : Exemples de crédits : la validation des modules représente 136 jours, 127 jours, 106 jours (sur les 146 jours requis) respectivement pour les formations maintenance aéronautique, avionique, formation mécanique automobile.

$(* * *)$ : Les notes 1 et 2 (« très bien » et « bien ») apportent plus de crédits que les notes 3 et 4 (« satisfaisant » et « suffisant »).

\section{Spécificités des solutions retenues pour la licence catégorie B (niveau technicien)}

On retrouve pour cette catégorie $\mathrm{B}$ des caractéristiques nationales déjà énoncées pour la catégorie $\mathrm{A}$. Quelques différenciations existent néanmoins dans chacun des trois pays.

\section{France}

La formation socle pour la catégorie B, le bac pro, se situe à un niveau plus élevé que celle de la catégorie $\mathrm{A}$ (CAP). Par ailleurs, il n'a pas été possible d'aménager le contenu de la formation spécifique existante à volume constant, comme cela a été le cas pour le CAP. Un complément de formation a été rajouté, prenant la forme d'une mention complémentaire (MC). La 
validation globale de la formation spécifique d'ancrage (le bac pro), en termes de crédits, a néanmoins permis de limiter à un an la durée du complément de formation requis.

Les candidats ayant obtenu le bac pro aéronautique mais n'ayant pas suivi ce complément de formation, peuvent obtenir, via le système de crédits, une validation complète ou partielle de modules de la licence $\mathrm{B}^{6}$.

\section{Allemagne}

Contrairement au cas français, les formations spécifiques et connexes validées comme socle pour la licence de catégorie B appartiennent au même niveau que celles prises en compte pour la catégorie A. Elles font partie du système dual.

À propos des aménagements du contenu des formations donnant accès à cette catégorie $B$, après quelques expérimentations partielles, la modalité dominante est celle des compléments individualisés, à la carte, offerts par le marché en fonction du bagage de formation des candidats et en référence à la formation duale.

\section{Royaume-Uni (RU)}

Comme pour la catégorie A, aucune formation (ou élément de formation) n'est validable pour la licence B.

$\mathrm{Au}$ spectre des formations préparant à l'examen, il faut ajouter les «foundation degrees », formations du supérieur, de type bac +2 , insérées dans les filières conduisant au «bachelor degree » (niveau $\mathrm{bac}+3)$.

En matière d'aménagement du contenu des formations, on retrouve les deux modalités en vigueur pour la catégorie A : création intégrale d'une formation de base Part-66 dans les écoles spécialisées, adaptations partielles pour des formations plus propédeutiques.

\footnotetext{
${ }^{6}$ Les titulaires du bac pro aéronautique bénéficient d'une validation complète pour les deux modules «mathématiques» et «aérodynamique » de la licence et d'une validation partielle pour cinq autres modules. Six modules de la licence sont par contre passer complètement. Sur les 670 questions d'origine, il en reste 390 à traiter. La détention du bac pro conduit donc à une réduction de 280 questions. Source : GSAC (Groupement pour la sécurité de l'aviation civile), Fascicule 55-17, Examens de conversion partie 66, applicables aux détenteurs de diplômes et/ou titres nationaux, 2005
}

Les «foundation degrees» jouissent d'un certain succès, d'une part parce que les étudiants qui s'inscrivent dans ce cycle bénéficient du système de bourses, et d'autre part parce que ces formations préparent sur une période courte (relativement à des parcours individualisés) à la licence. En France, cette démarche s'apparente à celle des élèves de BTS MEMA (Brevet de technicien supérieur de maintenance et exploitation de matériel aéronautique), qui se présentent à l'examen de la catégorie B.

\section{Des pratiques nationales affirmées}

L'analyse comparée des solutions nationales, en référence aux cinq critères retenus, permet de différencier les trois pays sur quelques points particuliers : le rôle de l'État central dans la recherche et la négociation d'une solution, la fonction assignée au système éducatif national, le degré d'intégration des filières de formation de base conduisant aux deux niveaux de licence $\mathrm{A}$ et $\mathrm{B}$.

\section{Un État plus ou moins dominant dans le processus de transposition}

En France, l'État central prend les initiatives sur la base d'une démarche concertée entre les ministères des Transports (tutelle de l'autorité de l'aviation civile) et de l'Éducation nationale, avec délégation de compétence matérialisée par la signature d'une convention de coopération.

La démarche est en revanche décentralisée et mixte en Allemagne. Si l'État fédéral, représenté par l'autorité de l'aviation civile, fournit un cadre général, les partenaires gestionnaires de la formation professionnelle négocient ensuite des solutions pour satisfaire ce cadre. La mixité se lit notamment dans la codétermination, par l'autorité et Lufthansa, des crédits (en termes de certification) octroyés aux formations existantes. L'intégration de cursus complémentaires dans les écoles en est une autre illustration : ces cursus ont été initiés par les écoles professionnelles, définis en coopération avec les entreprises locales, approuvés par l'autorité de l'aviation civile et financés par le ministère de l'Éducation au niveau régional (Land).

Au Royaume-Uni, elle est décentralisée et privée, du ressort du marché. Des organismes de formation 
privés procèdent, en fonction d'opportunités qu'ils sont seuls à apprécier, à des agencements particuliers de modules recouvrant partiellement ou entièrement les standards européens.

\section{Des postures}

des systèmes éducatifs nationaux différentes

Dans le cas de la France, on peut considérer le ministère de l'Éducation nationale comme un concessionnaire du chantier d'implantation de la Part-66. Corollaire du poids historique de l'État en matière d'éducation, le ministère de l'Éducation nationale s'est vu attribuer la gestion du dispositif (audits dans ses propres établissements et ceux qui sont sous sa tutelle, voie scolaire et apprentissage), contre l'attribution d'un large segment de marché et la prise en charge des frais de formation.

Dans le cas de l'Allemagne, l'appareil éducatif est davantage dans une posture de fournisseur. Par le jeu des crédits négociés avec l'autorité, l'appareil éducatif, déjà fortement professionnalisé, s'est attaché, dès le début du processus, à faire valider ses « produits » de base.

Au Royaume-Uni enfin, les aménageurs/constructeurs de formation sont assimilables à des entrepreneurs ayant à construire une offre marchande dont le contrôle qualité reste de la compétence de l'autorité de l'aviation civile, qui organise et gère la certification.

Les carences du système éducatif entre la fin de l'école obligatoire et le supérieur expliquent en partie le choix de cette solution. Au Royaume-Uni, une validation des formations n'a pu se faire car les entreprises et les centres de formation ne parvenaient pas à satisfaire les demandes de qualification en raison du coût élevé des investissements requis pour le montage de formations pointues dédiées à de très petits flux. En outre, le CAA (Civil Aviation Authority, autorité de l'aviation civile au RU) a mis en avant la faiblesse du niveau des sortants au palier GCSE (General Certificate of Secondary Education) en fin de premier cycle du secondaire, comme vivier potentiel des formations préparant à la licence, puisque ce sont essentiellement des sortants à ce niveau qui devraient s'inscrire dans des cycles de formation préparant à une certification Part-66.

\section{Des filières $d^{\prime}$ accès plus ou moins imbriquées}

Dans le cas français, les voies d'accès à chacune des deux catégories sont vraiment distinctes. Les formations «socle» pour la greffe des licences Part-66 appartiennent à des niveaux différents de formation, $\mathrm{V}$ et IV ${ }^{7}$, respectivement Certificat d'aptitude professionnelle (CAP) d'une part, Bac pro + Mention complémentaire (MC), d'autre part. Il y a étanchéité entre les deux voies.

En Allemagne, le même brevet dual sert d'ancrage pour les deux catégories $\mathrm{A}$ et $\mathrm{B}$, et constitue, en pratique, le point de départ d'une filière promotionnelle (brevet dual, licence A - licence B), la différence entre A et $\mathrm{B}$ s'exprimant dans le volume plus ou moins important des compléments de formation nécessaires.

Le Royaume-Uni se caractérise par une forte variété de parcours individuels et un dualisme dans la structuration des trajectoires dans des centres 147. Il existe en effet deux filières pour la préparation aux licences A et B :

- une première filière (filière bimodale) consiste en une inscription payante, soit à titre individuel soit dans le cadre d'une prise en charge par l'entreprise, pour la préparation d'une licence A ou d'une licence $\mathrm{B}$, les deux cursus étant bien distincts et non intégrés. Ces formations sont souvent proposées par des centres d'entreprise comme celui de la compagnie d'aviation $\mathrm{KLM} / \mathrm{UK}$, très prisé.

- une deuxième filière (promotionnelle) consiste à s'inscrire dans un cursus de formation intégrant les deux niveaux (un an pour la licence $A$ et ensuite un an pour la licence B); on la rencontre dans des centres annexés aux écoles publiques ou privées agréées Part-147 comme à Bristol ou à Perth.

Ces constats sur l'évolution de chacun des trois pays confrontés à une même contrainte externe peuvent s'interpréter en référence aux analyses de type « dépendance de sentier» (path dependency). Appliquées à plusieurs domaines (économie de l'innovation, gouvernance d'entreprise, etc.), ces analyses

\footnotetext{
7 Pour la nomenclature française de référence, le niveau V correspond à des formations et/ou des certifications normalement destinées à des candidats aux emplois d'ouvriers et d'employés qualifiés. Le niveau IV correspond à des formations et/ou des certifications normalement destinées à des candidats aux emplois de techniciens.
} 
mettent en exergue l'importance du contexte dans lequel chaque modèle est enraciné. La réussite de la transposition de dispositifs ou d'objectifs extérieurs à ce contexte passe obligatoirement par l'ancrage sur les règles et principes préexistants.

En l'occurrence, la formule des crédits, en permettant de valider une part plus ou moins grande des dispositifs nationaux de formation quand ils existent, contribue largement au résultat observé. Les pays ont effectivement cherché, dés le départ, à s'appuyer sur leurs propres dispositifs, soit pour y greffer les nouvelles dispositions européennes (Allemagne, France), soit pour créer une offre marchande de formations ciblées (Royaume-Uni).

L'Allemagne et la France se sont largement adossées à leurs systèmes publics de formation professionnelle initiale, qui offrent des cycles déjà bien structurés, pour une greffe des modules européens des licences A et B. Par ailleurs, historiquement, chacun de ces deux systèmes fonctionne sur la base d'instances de concertation pour la construction ou l'aménagement de l'offre de formation, auxquelles sont associés les partenaires concernés par les questions de formation et d'emploi ; ce fonctionnement accorde une moindre place qu'au Royaume-Uni aux ajustements par le marché notamment.

Reste qu'au-delà de ces grands traits qui renvoient aux structures organisationnelles nationales, d'autres observations nuancent quelque peu ce constat.

\section{Quelques restrictions aux effets structurels}

Il convient d'évoquer des observations qui pondèrent quelque peu les interprétations fondées sur le «tout structurel » : on assiste en effet à des différenciations au sein même des pays, et quelques solutions paraissent plutôt d'ordre conjoncturel.

Dans le cas de la France, l'Éducation nationale n'est pas le concessionnaire exclusif. Un nombre croissant d'établissements privés organise et gère également des formations de base aux licences. Des entreprises privées font en effet agréer leurs centres pour la formation de base donnant accès aux licences dans chacune des deux catégories.
Une différenciation existe également en Allemagne entre écoles pionnières et retardataires par rapport à l'adoption des normes 147 . On a observé effectivement que plusieurs écoles ont très tôt cherché à intégrer les modules Part-66 afin de faire valider rapidement leur offre de formation de base à la licence A et d'obtenir l'agrément Part-147, pour le plus grand avantage concurrentiel de leurs élèves. En effet, en raison de l'accréditation de l'établissement, ils bénéficient d'une réduction de la période pratique à l'issue de la formation de base. Ils pourront ainsi valoriser plus tôt, sur le marché du travail, leur certification complète Part-66 ( $c f$. encadré 1). Ces établissements pionniers se situent dans les grands centres aéronautiques régionaux, dans le Land de Hambourg notamment, sous la pression des professionnels (Airbus en particulier) qui apportent un soutien financier pour un aménagement rapide des formations. La rivalité Nord/Sud et la concurrence Hambourg/Munich ont fait le reste, les écoles de la région de Munich se sont empressées d'emboîter le pas aux initiatives précoces de leurs homologues du Nord.

Au Royaume-Uni, l'homogénéité n'est pas davantage de mise. Il existe une différenciation entre des solutions de formations intégrées dans des filières plus "propédeutiques» comme les "foundation degrees » insérées dans des cursus universitaires et débouchant sur le «bachelor degree », et des solutions exclusivement Part-66/147 préparant à l'exercice d'activités réglementées.

Une solution de nature conjoncturelle porte notamment sur le «spectre des formations » retenu pour une validation. Par crainte de pénuries de main-d'œuvre qualifiée, les partenaires en Allemagne - entreprises, Autorité nationale, écoles professionnelles - ont ainsi défini un large spectre de formations recevables pour un agrément partiel ou total Part-66. Les professionnels, et Lufthansa en particulier, ont plaidé en faveur de la prise en considération d'un grand nombre de formations. Derrière cette position, il y a également des considérations d'ordre socio-économique. En accroissant l'offre potentielle de licenciés et en considérant de fait la certification Part-66 comme le minimum requis pour l'exercice de la profession, le patronat limite ainsi les revendications salariales, les problèmes liés à une gestion trop rapide des carrières 
avec les conséquences sur la cohabitation entre générations.

Dans le cas de la France, a contrario, la faiblesse relative des besoins estimés a certainement joué en faveur d'une limitation du spectre des formations socle.

\section{DES PRÉOCCUPATIONS NATIONALES SPÉCIFIQUES}

La prise en considération des positions et stratégies d'acteurs est un complément utile à l'exposé des modalités de transposition des normes européennes. Elle aide à apprécier les processus, les débats nationaux, les concessions éventuelles qui ont conduit aux solutions retenues qui ne sont pas seulement des constructions techniques mais correspondent aussi à des compromis élaborés dans des contextes particuliers.

\section{France : obtenir des garanties pour les salariés}

Dès que le règlement européen Part-66 est devenu directement applicable (les dispositions du JAA devaient jusque-là être reprises par les réglementations nationales), les acteurs se sont impliqués dans la démarche d'intégration de la norme.

Des réunions ont été organisées sous l'égide de la DGAC, regroupant les acteurs concernés (les organisations syndicales, les associations, les entreprises de maintenance, les constructeurs, le GIFAS Groupement des industries françaises aéronautiques et spatiales etc.) afin de lever les ambiguïtés et/ou les contradictions contenues dans le texte proposé et de procéder à la réécriture des points litigieux.

Les débats sur la mise en œuvre des nouvelles normes ont porté essentiellement sur les garanties pour les salariés en poste. Il y a eu, par exemple, une définition des critères pour l'ordre d'urgence des conversions, avec un accord au niveau de la branche, pour faire passer en priorité les dossiers des salariés d'Air Lib. Ce transporteur aérien était en liquidation au moment de l'introduction des licences.
Côté syndical, si les grandes confédérations n'ont pas soutenu la création de la licence, elles se sont néanmoins interrogées sur l'attitude à adopter à son égard. Elles ont choisi d'aller à la négociation avec des revendications précises, sans chercher à bloquer le système, en s'attachant à défendre les intérêts des salariés. Elles ont tout d'abord plaidé pour une conception de la licence qui évite la division du personnel entre salariés très qualifiés et salariés peu qualifiés. Selon cette conception, les entreprises doivent former et perfectionner tous leurs salariés, sans distinction, les rendre tous éligibles à l'attribution de l'APRS et donc assurer à tous l'obtention de la licence. Initialement, les employeurs avaient en effet interprété le texte restrictivement, en considérant qu'ils devaient doter de licences uniquement ceux auxquels ils attribuent la responsabilité APRS.

Les syndicats estimaient que l'instauration de la licence correspondait à la reconnaissance que les individus possédaient bien les pré-requis en maintenance aéronautique. Ils ont ensuite largement mis en question l'article central 66.B.500 du règlement Part-66 traitant de la suspension et du retrait de la licence. Les expressions utilisées dans le texte de base sont en effet discutables. Comment peut-on, par exemple, affirmer, dans des circonstances particulières, qu'un agent peut « avoir fait preuve d'un entretien négligent »? Qui est habilité à porter un tel jugement? Quels sont les critères précis pour conclure de la sorte ? Des références à « la personne » ou encore à « l'organisme » donnaient lieu à contestations. Il fallait éviter qu'il puisse être fait un usage abusif du texte, au détriment des personnels.

Il reste encore des textes contestés par les syndicats, dont celui qui fait obligation au personnel de rapporter et signaler les défauts observés dans l'entreprise, mais aussi dans son environnement immédiat (la zone aéroportuaire pour la maintenance en ligne), ce texte étant inclus dans un cadre plus large de mesures de sécurité intérieure. Cette règle, qui existait déjà au Royaume-Uni, a été étendue à l'Europe. Le respect parfait d'une telle disposition étant impossible, les syndicats la considéraient comme porte ouverte à l'arbitraire le plus total. Dans le but d'écarter ce risque, ils imposaient une négociation nationale préalable à la mise en place de cette règle. Les représentants du patronat et des syndicats 
français interrogés avouent qu'il serait préférable dorénavant de s'impliquer le plus tôt possible dans le processus de construction des normes européennes, tant pour peser sur les choix arrêtés que pour envisager avec sérénité les solutions nationales possibles les plus avantageuses, sans avoir à subir la pression des échéances.

\section{L'Allemagne : réduire les coûts de formation pour les entreprises}

Dans ce pays, les débats ont essentiellement porté sur le choix entre d'une part la greffe des modules de la formation de base Part-66 sur le système dual existant et d'autre part la création d'une formation autonome. En effet, la formation exigée pour la licence A dure un peu moins d'un an, celle requise pour la licence B un peu moins de deux ans, alors que la durée de la formation duale est nettement plus longue (3,5 ans). Sachant qu'une année de formation duale aéronautique coûte relativement cher à l'entreprise, 50000 euros par apprenti, pourquoi la maintenir si la firme peut qualifier les personnels à moindre frais ?

Les compagnies s'exprimaient clairement dans le sens d'une réduction des coûts, en envisageant le remplacement de la formation duale par une formation moins coûteuse exclusivement Part-66. Les infrastructures et les compétences nécessaires pour gérer une telle formation existaient déjà dans des firmes comme Lufthansa.

Les acteurs de l'Éducation et l'autorité de l'aviation civile allemande avaient bien conscience de cette menace. L'Éducation professionnelle aurait alors perdu encore plus de renommée, de soutiens et d'élèves. L'autorité de l'aviation civile, quant à elle, craignait la disparition des effets bénéfiques d'une longue socialisation des jeunes en milieu professionnel via le système dual. La convergence des intérêts de ces deux acteurs pour la sauvegarde du système dual dans la maintenance aéronautique explique donc les traits majeurs de l'implantation de la Part-66 en Allemagne, la validation de la formation duale pour la Part-66 ( greffe»), l'aménagement de la formation duale, l'introduction d'un système de points de crédit.
Cette implantation est l'aboutissement d'une étroite coopération entre l'autorité et la Lufthansa, depuis la fin des années 90. Lufthansa a effectivement beaucoup d'expérience en matière d'ingénierie de formation. Elle dispose de spécialistes en la matière du fait de son implication dans les organes nationaux et régionaux (chambres consulaires) de pilotage et de gestion de la formation mécanique et aéronautique.

\section{Le Royaume-Uni : s'assurer de la qualité du système de licence européen}

$\mathrm{Au}$ Royaume-Uni, les débats ont porté essentiellement sur la difficulté, pour les négociateurs, à mettre en application la norme européenne au niveau du JAA et de l'AESA. L'accord pour le projet était fondé sur des principes d'égalité entre les pays membres et de respect par chacun d'un haut niveau de qualité. Les propos recueillis auprès de nos interlocuteurs, tant des syndicalistes que des représentants de l'autorité de l'aviation civile, dénoncent à la fois des pratiques nationales déviantes par rapport à ces principes et des dérogations trop facilement accordées aux États membres par l'Agence européenne de sécurité aérienne (AESA). On peut évoquer par exemple le différent entre le Royaume-Uni et la France à propos de la conversion des agents en poste avant l'introduction de la licence unifiée. Alors qu'au Royaume-Uni cette conversion se limitait aux agents déjà autorisés à délivrer l'APRS, la pratique en France, reflet de la politique déjà évoquée, élargissait ce cercle aux agents éligibles à cette autorisation.

Selon les représentants de l'autorité de l'aviation civile britannique questionnés, l'une des raisons majeures de ces incohérences résidait dans la concomitance de l'introduction de la licence et du transfert des responsabilités de surveillance à une agence européenne en création (l'AESA). Ce transfert aurait provoqué un «vide » temporel néfaste à la surveillance. L'un des interlocuteurs témoigne ainsi : "Au moment le plus crucial de l'instauration du système de licences, le processus de standardisation défini par le JAA a été enlevé et rien ne l'a remplacé. L'AESA est peu susceptible de pouvoir s'imposer à court terme, le vide qui en résulte permettant à quelques pays de faire des "interprétations" 
très larges des règles de mise en ouvre initialement convenues. Avant qu'AESA puisse s'imposer, les anomalies et les distorsions introduites pendant cette première période seront devenues un fait établi et par conséquent presque impossibles à rectifier " (entretien auprès de la Civil Aviation Authority).

Selon nous, la préoccupation accrue au RoyaumeUni pour la garantie de la qualité du dispositif est liée à la perception de la faiblesse du système national de formation professionnelle. L'autorité de l'aviation civile britannique est quasiment la seule en Europe à refuser d'emblée toute validation des formations nationales publiques, civiles et militaires, pour la licence. Contrairement à ses homologues européens, elle ne compte pas sur l'effet compensateur de ces formations au cas d'une fragilisation de la licence. Une telle situation - la licence perçue comme îlot de qualité isolé - explique pourquoi la difficulté européenne à conforter ce dispositif est devenue la question dominante au Royaume-Uni.

Dans les cas français et allemand, la solution de validation des formations initiales existantes, avec le système des crédits, est finalement satisfaisante, en termes de coûts d'adaptation, pour la collectivité et pour les entreprises en particulier. Les velléités de création de formations de branche, type CQP (Certificat de qualification professionnelle), tant en France qu'en Allemagne, ont été abandonnées au profit d'un recours aux appareils éducatifs nationaux.

Côté anglais, l'autorité de l'aviation civile admet qu'elle s'est battue pour le maintien de larges possibilités d'accès aux licences par le biais de l'expérience, en considérant les carences des pouvoirs publics en matière de formation professionnelle. Elle affirme par ailleurs avoir reçu l'adhésion des entreprises, des syndicats et des mécaniciens eux-mêmes, sur la base d'un militantisme, pour une licence telle qu'elle existait déjà en Grande-Bretagne.

L'acceptation des modalités de contrôle des connaissances sous forme de QCM (questionnaire à choix multiples), au nom de l'égalité et de la limitation des risques d'appréciations subjectives, est une des concessions de la France et de l'Allemagne, cette dernière ayant tenté en vain de s'opposer à la suppression des épreuves orales.
Concession aussi de la part des anglais qui plaidaient pour le maintien de ces épreuves, surtout revendiqué par les plus anciens qui ont dû les subir au prix d'efforts importants. Une autre concession importante est l'instauration de qualifications de type (type d'avion, type de moteur, type d'équipement), gérées par l'autorité nationale de l'aviation civile, ce qui « $a$ enlevé un grand degré de flexibilité des organisations JAR-145 et a accru la charge de l'Autorité avec des tâches bureaucratiques inutiles et très lourdes" (entretien auprès de la Civil Aviation Authority).

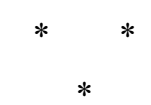

La présentation des modalités de mise en œuvre des Parts dans les trois pays montre que les solutions allemande et française ont pris appui sur les appareils nationaux respectifs de formation professionnelle initiale, en préservant les caractéristiques fondamentales de leurs certifications nationales : l'aspect très professionnalisant du brevet dual en Allemagne, la double finalité des diplômes en France (à la fois propédeutiques et professionnalisants). Dans ce dernier cas, la convention signée entre la DGAC et l'Éducation nationale reflète la «forte tradition de délégation des missions de formation initiale au système scolaire [...] ce qui limite la capacité d'expertise de la sphère productive dans les domaines de la formation [...]. De ce point de vue, le dispositif de formation français se trouve inscrit sur un sentier de dépendance qu'il sera difficile d'infléchir » (Le Bianic, 2000). Cet auteur met également en exergue l'effet «niveau de formation», caractéristique de la France. Cet effet a conduit, lors de la mise en oeuvre des Parts, à distinguer deux niveaux, $\mathrm{V}$ (Certificat d'aptitude professionnelle) et IV (bac pro) respectivement, pour l'ancrage des formations de base aux deux catégories de licence, A pour les postes d'ouvriers et B pour les postes de techniciens. Dans le cas de l'Allemagne, le corpus de formations de base commun aux deux catégories est le système dual.

Au Royaume-Uni, la solution a consisté à combiner des offres de formation de nature très différente. Des organismes de formation ont répondu aux besoins de professionnalisation à court terme sur un marché 
concurrentiel en offrant des formations qui reproduisent intégralement le standard européen. Des institutions universitaires publiques comme Kingston ont greffé des éléments de ce standard sur des formations spécialisées en aéronautique et intégrées dans des cursus comportant une dimension propédeutique.

La mise en œuvre des normes européennes traduit donc à la fois le poids des structures nationales dans le choix des solutions et la distribution traditionnelle des rôles entre les acteurs dans leur construction. En France, on retrouve la forte prégnance de l'État; en Allemagne, le rôle clé des professionnels et des organisations de branche (les « gouvernements privés ») ; au Royaume-Uni, la place faite au marché, typique du mode de régulation.

Une analyse approfondie des phénomènes à l'œuvre dans chacun des trois pays a néanmoins permis d'atténuer quelque peu les effets attribuables à ces structures nationales fortes. Des différenciations existent au sein même de chacun des pays. On a pu identifier par ailleurs l'impact des évènements conjoncturels sur les solutions adoptées.

Cependant, si quelques concessions ont été faites ici ou là afin de satisfaire aux obligations communautaires, elles ne semblent pas de nature à remettre fondamentalement en question les «régimes nationaux » (Soskice et Hancké, 1997) ${ }^{8}$.

Sans que l'on puisse parler de convergence des systèmes nationaux, peut-on avancer, néanmoins, à l'instar de Münk (2003), l'idée d'un rapprochement de ces systèmes ? Ou bien au contraire les pressions externes contribuent-elles au renforcement des particularismes?

Ces pressions externes, telles que l'imposition de normes supranationales, ont effectivement des effets, que l'on pourrait le cas échéant considérer comme positifs, pour une nation. Dans le cas anglais par exemple, la réglementation européenne sur le mode de production et d'usage des qualifications chez les mécaniciens avion a permis aux acteurs de la branche de prendre la mesure des déficits chroniques en

\footnotetext{
${ }^{8}$ On rappelle que, selon ces auteurs, la valeur que les acteurs économiques attribuent aux certifications de formation et d'enseignement dépend des modalités nationales d'articulation (qualifiées de " régime ») entre plusieurs dimensions, dont la plus ou moins forte structuration de l'appareil national de formation professionnelle.
}

matière de formation professionnelle initiale dans le pays. Le parlement a même été saisi du problème. Des rapports ont été produits sur la situation du marché du travail et sur les problèmes de qualification de la main-d'œuvre susceptibles d'avoir des conséquences néfastes dans un secteur stratégique pour le pays. Plus généralement (au-delà du cas de la maintenance, qui finalement bénéficierait de ce mouvement), des dispositions sont prises pour pallier les carences du système de formation initiale, tant générale que professionnelle, notamment au niveau technicien. Un dispositif de formation pour les jeunes de 14 à 19 ans est mis en place afin de structurer et d'accroître l'offre de formation au-delà du premier cycle du secondaire, augmenter le niveau des connaissances de base des formés, conduire un plus grand nombre vers le supérieur ${ }^{9}$. Un système de diplômes, accessibles par des voies de formation différenciées, a été conçu et organisé en référence à un cadre national des qualifications. Des modifications institutionnelles ont eu lieu afin de stimuler la concertation entre partenaires dans les différents secteurs d'activité lors de la construction de l'offre de formation. On pourrait ainsi conclure à une transposition de nombre de composantes des appareils éducatifs et de formation de pays voisins.

Force est d'admettre que des tendances sont à l'œuvre qui joueraient dans ce sens ; on peut citer les démarches de construction et de reconnaissance internationale des diplômes ${ }^{10}$, ou encore l'insertion des systèmes éducatifs nationaux dans des contextes

\footnotetext{
9 14-19 Education and Skills - White Paper Presented to Parliament by the Secretary of State for Education and Skills. February 2005. Document de consultation pré-législatif pour les parlementaires.

10 À l'exemple de l'initiative prise par la France, en 2001, connue sous le nom de «professionnalisation durable " (Asseraf, 2004), de construction de diplômes européens. Un groupe de travail intergouvernemental composé, pour chaque État participant (au départ la France, l'Allemagne, l'Espagne, la Grèce et le Royaume-Uni), de représentants des pouvoirs publics, d'experts en qualification et de représentants des branches professionnelles concernées, s'est régulièrement réuni depuis septembre 2001. La Commission européenne et le Cedefop (Centre européen de développement de la formation professionnelle) ont participé à ces travaux. Une maquette complète a d'abord été élaborée pour deux diplômes, un baccalauréat professionnel automobile et un brevet de technicien supérieur dans l'hôtellerie (Petitcolas, 2005). D'autres diplômes ont été construits par la suite. Les diplômes, validés par chaque pays, restent des diplômes nationaux, mais ils ont vocation, grâce aux référentiels communs, à être sinon reconnus d'office, au moins connus par les différents pays participants, acquérant ainsi, potentiellement, une valeur européenne. Depuis, l'initiative a été intégrée dans le programme européen Leonardo ayant pour objectif d'appuyer et de compléter les actions engagées par les États membres, grâce à un soutien à des projets de coopération transnationale dans le domaine de la formation professionnelle.
} 
européens, dans des marchés du travail transfrontaliers comme dans le cas des Parts. La «politique européenne de la formation professionnelle » deviendrait de plus en plus un élément de l'environnement de ces systèmes (Hanf, 1998, cité par Münk). Sans que soit mis en question le «noyau conceptuel» de chacun d'entre eux (noyau qui s'appuie sur les valeurs culturelles et historiques de chaque pays), un processus d'information mutuelle existerait malgré tout, qui peut servir la collectivité européenne. Ceci serait d'un intérêt certain eu égard au constat, opéré à l'occasion de l'étude sur l'application des règlements communautaires JARs/Part-66, de la défiance mutuelle entre acteurs dans différents pays, due pour partie à une méconnaissance patente des systèmes voisins.

\section{Bibliographie}

Asseraf G. (2004), « Propositions pour l'organisation d'un cadre commun de l'enseignement supérieur en Europe. Projet européen de "professionnalisation durable", vers un système de certification homogène pour les métiers ", in Actualité de la formation permanente, $\mathrm{n}^{\circ} 190$, mai-juin.

Charraud A.-M. (2002), «La construction de la certification: quelles méthodes, pour quels usages? » Journées de travail DESCO-CEREQ, Paris 13 et 14 septembre 2001, CPC documents, p. 150.

David P.-A. \& Foray D. (1995), « Dépendance du sentier et économie de l'innovation : un rapide tour d'horizon ", in Revue d'Economie Industrielle, $\mathrm{n}^{\circ}$ spécial, pp. 27-52.

Haas J. (2006), “Occupational Licensing versus Company-led Training. The Controversy over the Competence Assurance System for European Aircraft Technicians", Contribution au $16^{\mathrm{e}}$ Congrès mondial de l'Association internationale de sociologie (ISA), Durban, Afrique du Sud, du 23 au 29 juillet 2006.

Haas J. et Ourtau M. (2006), « De la réglementation nationale au certificat européen : tensions et solutions pour trois pays (Allemagne, France, RoyaumeUni): Le cas de la maintenance aéronautique », DESCO, CPC documents n ${ }^{\circ}$ 2006/4.
Le Bianic Th. (2000), Le dispositif français de formation professionnelle à l'épreuve des modèles allemand et britannique, Rapport de recherche, Lest/ CNRS, Aix-en-Provence.

Maurice M., Sellier F., Silvestre J.-J. (1982), Politique d'éducation et organisation industrielle en France et en Allemagne, Paris, PUF.

Méhaut Ph. (1997), "Le diplôme, une norme multivalente? », in Les diplômes professionnels en Allemagne et en France. Conception et jeux d'acteurs, Möbus M., Verdier E. (Ed.), L'Harmattan, novembre.

Möbus M. et Verdier E. (Éditeurs) (1997), Les diplômes professionnels en Allemagne et en France. Conception et jeux d'acteurs, L'Harmattan, novembre.

Münk D. (2003), «Les influences de 1'Union européenne sur le système de formation professionnelle en Allemagne ", Revue européenne Formation Professionnelle, $\mathrm{n}^{\circ} 30$, Cedefop, septembre-décembre, pp. 44 à 58 .

Petitcolas Ch. (2005), « Le point sur... Le BTS Responsable d'hébergement à référentiel commun européen, un BTS original », CPC Info, $\mathrm{n}^{\circ} 40$, premier semestre, pp. 49 à 51. 
Soskice D. et Hanke B. (1997), « De la construction des normes industrielles à l'organisation de la formation professionnelle. Une approche comparative», in Les diplômes professionnels en Allemagne et en France. Conception et jeux d'acteurs, Möbus M., Verdier E. (Ed.), L'Harmattan, novembre.

\title{
Résumé
}

\section{Certification européenne : rapprochement des structures nationales de formation?}

\author{
Joachim Haas et Maurice Ourtau
}

Afin de favoriser la libre circulation des personnes, la Communauté européenne encourage la normalisation des qualifications dans certains secteurs d'activité. De nouvelles modalités de certifications s'imposent aux États membres sous forme de règlements européens. La transposition de ces règlements dépend des systèmes nationaux de production et de reconnaissance des qualifications qui ont leur propre cohérence. II est donc intéressant d'analyser ce processus de transposition dans des pays réputés posséder chacun des caractéristiques fortes en matière de formation et de certification. Cette contribution s'appuie sur l'étude du cas particulier des nouvelles certifications en vigueur dans le secteur de la maintenance aéronautique.

\section{Mots-clés}

Certification, Communauté européenne, maintenance industrielle, industrie aéronautique, politique de formation

Journal of Economic Literature : I 28, J 24 


\section{Horizons stratégiques}

Revue trimestrielle du Centre d'analyse stratégique

\section{N 6 - octobre 2007 - Numéro spécial Informer sur l'Europe en France}

\section{DOSSIER}

- Introduction: Quelles stratégies d'information en France sur l'Europe?

(Thierry Chopin et Marie-Cécile Milliat)

\section{DÉBAT}

- avec Sylvie Goulard, Pierre Lequiller et Jean Quatremer

\section{FOCUS}

- Les Français et leur connaissance de l'Europe

(Bruno Cautrès)

- Enseigner l'Europe ? L'expérience du manuel franco-allemand d'Histoire (Étienne François)

- La professionnalisation du travail associatif autour de l'information européenne :

le rôle des Maisons de l'Europe

(Julien Weisbein)

- L'évolution des structures d'information consacrées à l'Union européenne

sur Internet : l'expérience d'EurActiv

(Nathalie Lhayani)

\section{PERSPECTIVES INTERNATIONALES}

- Les médias et l'Union européenne : le prisme national perdure

(Gerd G. Kopper)

- Rediscovering Europe in The Netherlands: Towards Strengthened Legitimacy of Dutch EU Policy (Ton van den Brink et Mendeltje van Keulen)

\section{ANALYSE}

- Les groupes d'intérêt, vecteurs d'information sur l'Europe?

(Mathilde Durand et Hélène Jorry)

- Europe : le devoir d'informer, le devoir de comprendre. Éléments d'une stratégie publique pour la France (François-Xavier Priollaud)

- La Présidence française et les élections européennes : de la communication institutionnelle

à l'information politique ?

(Yves Bertoncini et Thierry Chopin)

\section{CHRONIQUES}

- Actualité des homologues étrangers (Aude Rousselot)

- Échos (Comptes-rendus de colloques et publications ; Analyses critiques)

- Actualités du Centre d'analyse stratégique

- Résumés - Summaries - Resúmenes - Zusammenfassungen

- Notices biographiques 